\title{
Horoball packings and their densities by generalized simplicial density function in the hyperbolic space *
}

\author{
Jenő Szirmai \\ Budapest University of Technology and Economics \\ Institute of Mathematics, Department of Geometry \\ H-1521 Budapest, Hungary \\ Email: szirmai@math.bme.hu
}

(October 3, 2018)

\begin{abstract}
The aim of this paper is to determine the locally densest horoball packing arrangements and their densities with respect to fully asymptotic tetrahedra with at least one plane of symmetry in hyperbolic 3-space $\overline{\mathbf{H}}^{3}$ extended with its absolute figure, where the ideal centers of horoballs give rise to vertices of a fully asymptotic tetrahedron. We allow horoballs of different types at the various vertices. Moreover, we generalize the notion of the simplicial density function in the extended hyperbolic space $\overline{\mathbf{H}}^{n},(n \geq 2)$, and prove that, in this sense, the well known Böröczky-Florian density upper bound for "congruent horoball" packings of $\overline{\mathbf{H}}^{3}$ does not remain valid to the fully asymptotic tetrahedra.

The density of this locally densest packing is $\approx 0.874994$, may be surprisingly larger than the Böröczky-Florian density upper bound $\approx 0.853276$ but our local ball arrangement seems not to have extension to the whole hyperbolic space.
\end{abstract}

*Mathematics Subject Classification 2010: 52C17, 52C22, 52B15.

Key words and phrases: Hyperbolic geometry, horoball packings, optimal simplicial density. 


\section{Basic notions}

\subsection{Local density function}

We summarize the most important definitions and results about ball packings in $\overline{\mathbf{H}}^{n}(n \geq 2)$. For more details and proofs, we refer to [1], [4], [8], [9] and [16]. There are different notions of packing density. For later purposes, the local density measure is the best suited one. In the $n$-dimensional $(n \geq 2)$ hyperbolic space there are 3-types of spheres: sphere, horosphere and hypersphere. Now, we consider the horospheres and their bodies, the horoballs. A horoball packing $\mathcal{B}_{h}=\left\{B_{h}\right\}$ of $\overline{\mathbf{H}}^{n}$ is an arrangement of non-overlapping horoballs $B_{h}$ in $\overline{\mathbf{H}}^{n}$. The notion of local density of the usual ball packing can be extended for horoball packings $\mathcal{B}_{h}$ of $\overline{\mathbf{H}}^{n}$. Let $B_{h} \in \mathcal{B}_{h}$, and $P \in \overline{\mathbf{H}}^{n}$ an arbitrary point. Then, $\rho\left(P, B_{h}\right)$ is defined to be the length of the unique perpendicular from $P$ to the horosphere $S_{h}$ bounding $B_{h}$, where again $\rho\left(P, B_{h}\right)$ is taken negative for $P \in B_{h}$. The DirichletVoronoi cell (shortly D-V cell) $\mathcal{D}\left(B_{h}\right)$ of $B_{h}$ in $\mathcal{B}_{h}$ is defined to be the convex body

$$
\mathcal{D}_{h}=D\left(\mathcal{B}_{h}, B_{h}\right):=\left\{P \in \mathbf{H}^{n} \mid \rho\left(P, B_{h}\right) \leq \rho\left(P, B_{h}^{\prime}\right), \forall B_{h}^{\prime} \in \mathcal{B}_{h}\right\} .
$$

Since both, $B_{h}$ and $\mathcal{D}_{h}$, are of infinite volume, the usual concept of local density has to be modified. Let $Q \in \partial \mathbf{H}^{n}$ denote the base point (ideal center at the infinity) of $B_{h}$, and interpret $S_{h}$ as a Euclidean $(n-1)$-space. Let $B_{n-1}(R) \subset S_{h}$ be an $n$-1-ball with center $C \in S_{h}$. Then, $Q \in \partial \mathbf{H}^{n}$ and $B_{n-1}(R)$ determine a convex cone $C_{n}(R):=\operatorname{cone}\left(B_{n-1}^{Q}(R)\right) \in \overline{\mathbf{H}}^{n}$ with apex $Q$ consisting of all hyperbolic geodesics through $B_{n-1}(R)$ with limiting point $Q$. With these preparations, the local density $\delta_{n}\left(B_{h}, \mathcal{B}_{h}\right)$ of $B_{h}$ to $\mathcal{D}_{h}$ is defined by

$$
\delta_{n}\left(\mathcal{B}_{h}, B_{h}\right):=\varlimsup_{R \rightarrow \infty} \frac{\operatorname{vol}\left(B_{h} \cap C_{n}(R)\right)}{\operatorname{vol}\left(\mathcal{D}_{h} \cap C_{n}(R)\right)}
$$

and this limes superior is independent of the choice of the center $C$ of $B_{n-1}(R)$.

\subsection{Densest packings with horoballs of the same type}

We have to change the notion of the ,congruent” horoballs in a horoball packing to the horoballs of the "same type" because the horroballs are congruent in the hyperbolic space $\overline{\mathbf{H}}^{n}$. Two horoballs in a horoball packing are in the "same type" if and only if the local densities of the horoballs to the corresponding cell (e.g. 
$D$-V cell; or ideal simplex, later on) are equal. If we assume that the „horoballs belong to the same type", then by analytical continuation, the well known simplicial density function on $\overline{\mathbf{H}}^{n}$ can be extended from $n$-balls of radius $r$ to the case $r=\infty$, too. Namely, in this case consider $n+1$ horoballs $B_{h}$ which are mutually tangent. The convex hull of their base points at infinity will be a totally asymptotic or ideal regular simplex $T_{\text {reg }} \in \overline{\mathbf{H}}^{n}$ of finite volume. Hence, in this case it is legitimate to write

$$
d_{n}(\infty)=(n+1) \frac{\operatorname{vol}\left(B_{h}\right) \cap T_{\text {reg }}}{\operatorname{vol}\left(T_{\text {reg }}\right)} .
$$

Then for a horoball packing $\mathcal{B}_{h}$, there is an analogue of ball packing, namely (cf. [4], Theorem 4)

$$
\delta_{n}\left(\mathcal{B}_{h}, B_{h}\right) \leq d_{n}(\infty), \forall B_{h} \in \mathcal{B}_{h} .
$$

The upper bound $d_{n}(\infty)(n=2,3)$ is attained for a regular horoball packing, that is, a packing by horoballs which are inscribed in the cells of a regular honeycomb of $\overline{\mathbf{H}}^{n}$. For dimensions $n=2$, there is only one such packing. It belongs to the regular tessellation $\{\infty, 3\}$. Its dual $\{3, \infty\}$ is the regular tessellation by ideal triangles all of whose vertices are surrounded by infinitely many triangles. This packing has in-circle density $d_{2}(\infty)=\frac{3}{\pi} \approx 0.95493 \ldots$

In $\overline{\mathbf{H}}^{3}$ there is exactly one horoball packing whose Dirichlet-Voronoi cells give rise to a regular honeycomb described by the Schläfli symbol $\{6,3,3\}$. Its dual $\{3,3,6\}$ consists of ideal regular simplices $T_{\text {reg }}$ with dihedral angle $\frac{\pi}{3}$ building up a 6-cycle around each edge of the tessellation.

\subsection{Optimal packings by horoballs of different types in $\overline{\mathbf{H}}^{n}$}

In [9] we have determined the optimal horoball packing arrangements and their densities for all four fully asymptotic Coxeter tilings (Coxeter honeycombs) in $\overline{\mathbf{H}}^{3}$. Centers of horoballs are required to lie at vertices of the regular polyhedral cells constituting the tiling. We allow horoballs of different types at the various vertices. We have proved that the known Böröczky-Florian density upper bound for "congruent horoball" packings of $\overline{\mathbf{H}}^{3}$ remains valid for the class of fully asymptotic Coxeter tilings, even if packing conditions are relaxed by allowing horoballs of different types under prescribed symmetry groups. The consequences of this remarkable result are discussed for various Coxeter tilings (see [9]), but in this paper we consider only the tetrahedral Coxeter tilings. 


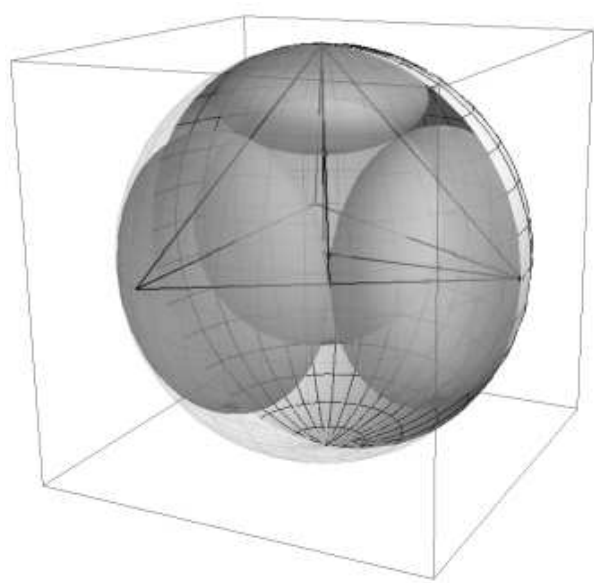

a.



b.

Figure 1: Two optimal horoball arrangemets of $(3,3,6)$ tiling.

The Coxeter tiling $\{3,3,6\}$ is a three-dimensional honeycomb with cells comprised of fully asymptotic regular tetrahedra. We arbitrarily choose such a tetrahedron $T_{\text {reg }}=E_{0} E_{1} E_{2} E_{3}$, and place the horoball centers at the ideal vertices $E_{0}, \ldots, E_{3}$. We vary the types of the horoballs so that they satisfy our constraints of non-overlap. The density of horoball packings to the above honeycomb can be computed by the new type of the simplicial density function:

Definition 1.1 The density of a horoball packing in Coxeter honeycomb $\{3,3,6\}$ is defined as

$$
\delta\left(\mathcal{B}_{336}\right)=\frac{\sum_{i=1}^{4} \operatorname{vol}\left(B_{i} \cap T_{\text {reg }}\right)}{\operatorname{vol}\left(T_{\text {reg }}\right)} .
$$

Theorem 1.2 There are two distinct optimally dense horoball arrangements $\mathcal{B}_{336}^{i}$, $(i=1,2)$ for the tetrahedral Coxeter tiling $(3,3,6)$ with the same density: $\delta\left(\mathcal{B}_{336}^{i}\right) \approx$ 0.85327609 .

Fig. 1. shows the two optimal horoball arrangements in Beltrami-Cayley-Klein model. 


\subsection{Generalization of the simplicial density function}

Definition 1.3 We consider an arbitrary fully asymptotic simplex $T=E_{0} E_{1}$ $E_{2} E_{3} \ldots E_{n}$ in the n-dimensional hyperbolic space $\overline{\mathbf{H}}^{n}$. Centers of horoballs are required to lie at vertices of $T$. We allow horoballs $\left(B_{i}, i=1,2, \ldots, n\right)$ of different types at the various vertices and require to form a packing, moreover we assume that

$$
\operatorname{card}\left(B_{i} \cap\left[E_{i_{0}} E_{i_{1}} \ldots E_{i_{n-1}}\right]\right) \leq 1, \quad i_{j} \neq i, \quad j \in\{0,1, \ldots, n-1\}
$$

(The hyperplane of points $E_{i_{0}}, E_{i_{1}}, \ldots, E_{i_{n-1}}$ is denoted by $\left[E_{i_{0}} E_{i_{1}} \ldots E_{i_{n-1}}\right]$ may touch the horoball $B_{i_{n}}$.) The generalized simplicial density function for the above simplex and horoballs is defined as

$$
\delta(\mathcal{B})=\frac{\sum_{i=0}^{n} \operatorname{vol}\left(B_{i} \cap T\right)}{\operatorname{vol}(T)} .
$$

For $n=3$ the main problem is to find a fully asymptotic tetrahedron $T_{\text {opt }} \in$ $\overline{\mathbf{H}}^{3}$ and horoballs $B_{i}$ centered at the vertices $E_{i}$ such that the density $\delta(\mathcal{B})$ (see Definition 1.3) of the corresponding horoball arrangement is maximal. In this case the horoball arrangement $\mathcal{B}$ is said to be locally optimal.

In this paper, we study locally optimal horoball packings for fully asymptotic tetrahedra in $\overline{\mathbf{H}}^{3}$, while allowing different types of horoballs to be centered at the vertices of the fully asymptotic tetrahedra.

The general investigation of this problem seems to be very difficult, thus we assume for simplicity that the tetrahedron has at least one plane of symmetry, simply called plane symmetric. So it will have only one free angle parameter. Then this ideal tetrahedron has 2 orthogonal symmetry planes and 2 symmetry lines of these planes, i.e. the symmetry group is of order 8 , denoted by $2{ }^{*} \mathbf{2}=\overline{4} \mathbf{m} 2$ (by Conway and Hermann-Mauguin, respectively).

\section{Computations in projective model}

For $\overline{\mathbf{H}}^{n} n \geq 2$ we use the projective model in Lorentz space $\mathbf{E}^{1, n}$ of signature $(1, n)$, i.e. $\mathbf{E}^{1, n}$ is the real vector space $\mathbf{V}^{n+1}$ equipped with the bilinear form of signature $(1, n)$

$$
\langle\mathbf{x}, \mathbf{y}\rangle=-x^{0} y^{0}+x^{1} y^{1}+\cdots+x^{n} y^{n}
$$


where the non-zero vectors

$$
\mathbf{x}=\left(x^{0}, x^{1}, \ldots, x^{n}\right) \in \mathbf{V}^{n+1} \text { and } \mathbf{y}=\left(y^{0}, y^{1}, \ldots, y^{n}\right) \in \mathbf{V}^{n+1},
$$

are determined up to real factors and they represent points in $\mathcal{P}^{n}(\mathbf{R}) . \mathbf{H}^{n}$ is represented as the interior of the absolute quadratic form

$$
Q=\left\{[\mathbf{x}] \in \mathcal{P}^{n} \mid\langle\mathbf{x}, \mathbf{x}\rangle=0\right\}=\partial \mathbf{H}^{n}
$$

in real projective space $\mathcal{P}^{n}\left(\mathbf{V}^{n+1}, \boldsymbol{V}_{n+1}\right)$. All proper interior point $\mathbf{x} \in \mathbf{H}^{n}$ are characterized by $\langle\mathbf{x}, \mathbf{x}\rangle<0$.

The points on the boundary $\partial \mathbf{H}^{n}$ in $\mathcal{P}^{n}$ represent the absolute points at infinity of $\overline{\mathbf{H}}^{n}$. Points $\mathbf{y}$ with $\langle\mathbf{y}, \mathbf{y}\rangle>0$ lie outside of $\overline{\mathbf{H}}^{n}$ and are called outer points of $\mathbf{H}^{n}$. Let $X([\mathbf{x}]) \in \mathcal{P}^{n}$ a point; $[\mathbf{y}] \in \mathcal{P}^{n}$ is said to be conjugate to $[\mathbf{x}]$ relative to $Q$ when $\langle\mathbf{x}, \mathbf{y}\rangle=0$. The set of all points conjugate to $X([\mathbf{x}])$ form a projective polar hyperplane

$$
\operatorname{pol}(X):=\left\{[\mathbf{y}] \in \mathcal{P}^{n} \mid\langle\mathbf{x}, \mathbf{y}\rangle=0\right\} .
$$

Hence the bilinear form $Q$ by (2.1) induces a bijection (linear polarity $\mathbf{V}^{n+1} \rightarrow$ $\left.\boldsymbol{V}_{n+1}\right)$ from the points of $\mathcal{P}^{n}$ onto its hyperplanes.

Point $X[\mathbf{x}]$ and the hyperplane $\alpha[\boldsymbol{a}]$ are called incident if the value of the linear form $\boldsymbol{a}$ on the vector $\mathbf{x}$ is equal to zero; i.e., $\mathbf{x} \boldsymbol{a}=0\left(\mathrm{x} \in \mathbf{V}^{n+1}\right.$ $\left.\{\mathbf{0}\}, \boldsymbol{a} \in \boldsymbol{V}_{n+1} \backslash\{\mathbf{0}\}\right)$. Straight lines in $\mathcal{P}^{n}$ are characterized by the 2-subspaces of $\mathbf{V}^{n+1}$ or $(n-1)$-spaces of $\boldsymbol{V}_{n+1}$ (see e.g. in [11]).

In this paper we set the sectional curvature of $\overline{\mathbf{H}}^{n}, K=-k^{2}$, to be $k=1$. The distance $s$ of two proper points $(\mathbf{x})$ and $(\mathbf{y})$ is calculated by the formula:

$$
\cosh s=\frac{-\langle\mathbf{x}, \mathbf{y}\rangle}{\sqrt{\langle\mathbf{x}, \mathbf{x}\rangle\langle\mathbf{y}, \mathbf{y}\rangle}} .
$$

The foot point $Y(\mathbf{y})$ of the perpendicular, dropped from the point $X(\mathbf{x})$ on the plane $(u)$, has the following form:

$$
\mathbf{y}=\mathbf{x}-\frac{\langle\mathbf{x}, \mathbf{u}\rangle}{\langle\mathbf{u}, \mathbf{u}\rangle} \mathbf{u}
$$

\subsection{On horospheres in hyperbolic space $\overline{\mathbf{H}}^{3}$}

Definition 2.1 A horosphere in the hyperbolic geometry is the surface orthogonal to the set of parallel lines, passing through the same point on the absolute quadratic surface ( simply absolute) of $\overline{\mathbf{H}}^{3}$. 
We represent $\overline{\mathbf{H}}^{3}$ by the Beltrami-Cayley-Klein ball model. We introduce a projective coordinate system using vector basis $\mathbf{b}_{i}(i=0,1,2,3)$ for $\mathcal{P}^{3}$ where the coordinates of the center of the model is $(1,0,0,0)$. We pick up an arbitrary point at infinity to be $E_{3}(1,0,0,1)$ (see Fig. 3).

We obtain the following equation for the horosphere in our Beltrami-CayleyKlein model of $\overline{\mathbf{H}}^{3}$ above:

$$
-2 s x^{0} x^{0}-2 x^{3} x^{3}+2(s+1)\left(x^{0} x^{3}\right)+(s-1)\left(x^{1} x^{1}+x^{2} x^{2}\right)=0
$$

Remark 2.2 1. We get the equation of the horosphere in the Cartesian coordinate $\operatorname{system}\left(x:=\frac{x^{1}}{x^{0}}, y:=\frac{x^{2}}{x^{0}}, z:=\frac{x^{3}}{x^{0}}\right)$ :

$$
\frac{2\left(x^{2}+y^{2}\right)}{1-s}+\frac{4\left(z-\frac{s+1}{2}\right)^{2}}{(1-s)^{2}}=1 \text {. }
$$

2. We will also use the equation of the horosphere with center $E_{2}$ (see Fig. 3):

$$
\frac{2\left(\frac{1}{4} x^{2}+y^{2}+\frac{3}{4} z^{2}+\frac{\sqrt{3}}{2} x z\right)}{1-s_{1}}+\frac{4\left(\frac{\sqrt{3}}{2} x-\frac{1}{2} z-\frac{s_{1}+1}{2}\right)^{2}}{\left(1-s_{1}\right)^{2}}=1 .
$$

The length $l(x)$ of a horocycle arc to a chord segment $x$ is determined by the classical formula due to J. Bolyai:

$$
l(x)=k \sinh \frac{x}{k} .
$$

The intrinsic geometry of the horosphere is Euclidean, therefore, the area $\mathcal{A}$ of a horospherical triangle will be computed by the formula of Heron. The volume of the horoball sectors can be calculated using another formula by J. Bolyai. If the area of a domain on the horoshere is $\mathcal{A}$, the volume determined by $\mathcal{A}$ and the aggregate of axes drawn from $\mathcal{A}$ is equal to

$$
V=\frac{1}{2} k \mathcal{A},
$$

we assume that $k=1$ in this paper. 


\subsection{The volume of the fully asymptotic tetrahedron in $\overline{\mathbf{H}}^{3}$}

A tetrahedron $T$ in hyperbolic space is determined in general by its six dihedral angles i.e. the mutual angles between the four faces of this tetrahedron. In order to calculate the volume $\operatorname{vol}(T)$ of $T$; one can dissect $T$ into six orthoschemes and their volumes can be calculated by the Lobachevsky formula.

A plane orthoscheme is a right-angled triangle, whose area can be expressed by the well known defect formula. For three-dimensional spherical orthoschemes, Ludwig Schläfli, about 1850, was able to find the volume differential depending on differentials of the not fixed 3 dihedral angles. Already in 1836, Lobachevsky found a volume formula for three-dimensional hyperbolic orthoschemes $\mathcal{O}$ [3].

The integration method for orthoschemes of dimension three was generalized by Böhm in 1962 [3] to spaces of constant nonvanishing curvature of arbitrary dimension.

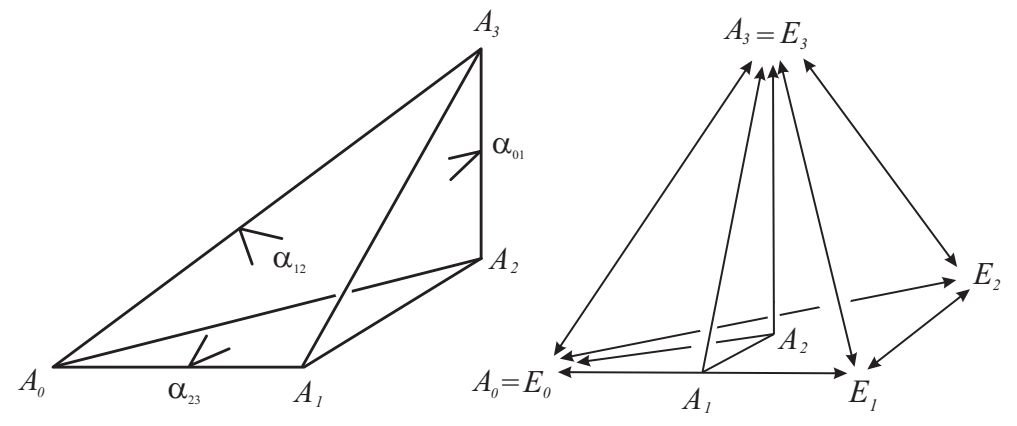

Figure 2:

Theorem 2.3 (N. I. Lobachevsky). The volume of a three-dimensional hyperbolic orthoscheme $\mathcal{O} \subset \overline{\mathbf{H}}^{3}$ is expressed with the dihedral angles $\alpha_{01}, \alpha_{12}, \alpha_{23}, \quad(0 \leq$ $\left.\alpha_{i j} \leq \frac{\pi}{2}\right)$ (Fig. 2) in the following form:

$$
\begin{aligned}
& \operatorname{vol}(\mathcal{O})=\frac{1}{4}\left\{\mathcal{L}\left(\alpha_{01}+\theta\right)-\mathcal{L}\left(\alpha_{01}-\theta\right)+\mathcal{L}\left(\frac{\pi}{2}+\alpha_{12}-\theta\right)+\right. \\
& \left.+\mathcal{L}\left(\frac{\pi}{2}-\alpha_{12}-\theta\right)+\mathcal{L}\left(\alpha_{23}+\theta\right)-\mathcal{L}\left(\alpha_{23}-\theta\right)+2 \mathcal{L}\left(\frac{\pi}{2}-\theta\right)\right\}
\end{aligned}
$$

where $\theta \in\left[0, \frac{\pi}{2}\right)$ is defined by the following formula:

$$
\tan (\theta)=\frac{\sqrt{\cos ^{2} \alpha_{12}-\sin ^{2} \alpha_{01} \sin ^{2} \alpha_{23}}}{\cos \alpha_{01} \cos \alpha_{23}}
$$


and where $\mathcal{L}(x):=-\int_{0}^{x} \log |2 \sin t| d t$ denotes the Lobachevsky function.

We obtain the volume formula for the asymptotical orthoschems if $A_{0}$ and $A_{3}$ are at the absolute i.e. $\alpha:=\alpha_{01}=\frac{\pi}{2}-\alpha_{12}=\alpha_{23}=\theta$ :

$$
\operatorname{vol}\left(\mathcal{O}_{\infty}\right)=\frac{1}{2} \mathcal{L}(\alpha)
$$

J. Milnor's volume formula of a fully asymptotic tetrahedron $T(\alpha, \beta)$ with dihedral angles $\alpha, \beta, \gamma$ is determined by its division into orthoschems (see [14]):

$$
\operatorname{vol}(T(\alpha, \beta))=\mathcal{L}(\alpha)+\mathcal{L}(\beta)+\mathcal{L}(\gamma), \text { where } \alpha+\beta+\gamma=\pi .
$$

As an easy consequence, we get the following

Lemma 2.4 The volume formula vol $(T(\alpha))$ of a fully asymptotic plane symmetric tetrahedron $T(\alpha)$ can be derived by the duplication law:

$$
\operatorname{vol}(T(\alpha))=2 \mathcal{L}(\alpha)-\mathcal{L}(2 \alpha)=-2 \mathcal{L}\left(\alpha+\frac{\pi}{2}\right) .
$$

In [8] there are further results for the volumes of orthoschemes and simplices in higher dimensions.

\section{Horoball packings for asymptotic tetrahedra}

The aim of this section is to determine the optimal packing arrangement and its densities for the fully asymptotic tetrahedra in $\overline{\mathbf{H}}^{3}$.

We will make heavy use of the following Lemma (see also [17]):

Lemma 3.1 Let $B_{1}$ and $B_{2}$ denote two horoballs with ideal centers $C_{1}$ and $C_{2}$ respectively. Take $\tau_{1}$ and $\tau_{2}$ to be two congruent trihedra, with vertices $C_{1}$ and $C_{2}$. Assume that these horoballs $B_{1}(x)$ and $B_{2}(x)$ are tangent at point $I(x) \in C_{1} C_{2}$ and $C_{1} C_{2}$ is a common edge of the two trihedra $\tau_{1}$ and $\tau_{2}$. We define the point of contact I(0) such that the following equality holds for the volumes of horoball sectors:

$$
V(0):=2 \operatorname{vol}\left(B_{1}(0) \cap \tau_{1}\right)=2 \operatorname{vol}\left(B_{2}(0) \cap \tau_{2}\right) .
$$

If $x$ denotes the hyperbolic distance between $I(0)$ and $I(x)$, then the function

$$
V(x):=\operatorname{vol}\left(B_{1}(x) \cap \tau_{1}\right)+\operatorname{vol}\left(B_{2}(x) \cap \tau_{2}\right)=\frac{V(0)}{2}\left(e^{2 x}+e^{-2 x}\right)
$$

strictly increases as $x \rightarrow \pm \infty$. 
We arbitrarily choose a fully asymptotic tetrahedron $T(\alpha)=E_{0} E_{1} E_{2} E_{3}$ with at least one plane of symmetry (see Section 1.4 and Fig. 3), and place the horoball centers at vertices $E_{0}, \ldots, E_{3}$. We vary the types of the horoballs so that they satisfy our constraints of non-overlap. The packing density is obtained by Definition 1.3. The dihedral angles of the above tetrahedron at the edges $E_{0} E_{1}, E_{0} E_{2}, E_{1} E_{3}, E_{2} E_{3}$ are denoted by $\alpha$ and it is clear that the dihedral angles at the remaining edges $E_{0} E_{3}$ and $E_{1} E_{2}$ are $\pi-2 \alpha$.

We introduce a Euclidean projective coordinate system to the tetrahedron $T(\alpha)$ by the following coordinates of the vertices:

$$
\begin{gathered}
E_{0}:=\left(1,0, \sqrt{1-z^{2}}, z\right),(-1 \leq z \leq 1) ; E_{1}=\left(1, \frac{\sqrt{3}}{2}, 0,-\frac{1}{2}\right) ; \\
E_{2}=\left(1,-\frac{\sqrt{3}}{2}, 0,-\frac{1}{2}\right) ; E_{3}:=(1,0,0,1) .
\end{gathered}
$$

$E_{0}$ lies on the symmetry plane $x=0$ of tetrahedron $T(\alpha)$ (see Fig. 3 ). In order to determine the optimal ball arrangement and its fully asymptotic tetrahedron first we consider the following situations:

1. In an optimally dense packing, the horoball packing must be locally stable, i.e. each ball is fixed by its neighboring horoballs or by the opposite face of the tetrahedron. Otherwise the density could be improved by blowing up at least one horoball until it touches a neighboring horoball or its opposite face of tetrahedron.

2. We fix the parameter $z(z \in(-1,1) \backslash 0)$ and blow up the horoballs $B_{i}$ centered at the vertices of $T(\alpha)$ so that the volumes $\operatorname{vol}\left(B_{i} \cap T(\alpha)\right),(i=$ $0,1,2,3)$ are equal until certain two neighbouring horoballs touch each other. In this situation we distinguish two cases for the varying vertex $E_{0}$ :

i.) If $0<z<1$ then the horoballs $B_{3}-B_{0}$ and $B_{1}-B_{2}$ touch each other the other horoballs do not.

ii.) If $-1<z<0$ then each of the horoball pairs $B_{0}-B_{1}, B_{0}-B_{2}, B_{1}-$ $B_{3}, B_{2}-B_{3}$ have exactly one common point, respectively, and $B_{3}-B_{0}$ and $B_{1}-B_{2}$ do not touch each other.

If $z=0$ the tetrahedron is regular $T(\alpha)=T_{\text {reg }},\left(\alpha=\frac{\pi}{3}\right)$ and each horoball touch all neigboring horoballs. This case is studied in details formerly in [9]. 
3. The volume $\operatorname{vol}(T(\alpha)$ is determined by Lemma 2.4, formula (2.12), and by the above machinery of hyperbolic geometry we obtain the following formulas:

$$
\begin{gathered}
\cos (2 \alpha)=-\frac{1+2 z}{z-2}, \quad(-1<z<1) \\
\operatorname{vol}(T(\alpha))=-2 \mathcal{L}\left[\frac{1}{2} \arccos \left(-\frac{1+2 z}{z-2}\right)+\frac{\pi}{2}\right] .
\end{gathered}
$$

i/1: $\quad\left(z \in\left(0, \frac{-2}{13}+\frac{6 \sqrt{3}}{13}\right]\right)$

a.) First, we define the tangent point $I(0) \in E_{0} E_{3}$ of horoballs $B_{0}(0)$ and $B_{3}(0)$ so that equalities hold for the volumes of all horoball sectors.

The volume sum of horoball sectors $B_{i}(0) \cap T(\alpha),(i=0,1,2,3)$ in $T(\alpha)$ is

$$
V(0)=\sum_{1}^{4}\left(\operatorname{vol}\left(B_{i}(0) \cap T(\alpha)\right)\right)=4 \operatorname{vol}\left(B_{1}(0) \cap T(\alpha)\right) .
$$

In the introduced coordinate system $B_{1}(0)$ and $B_{2}(0)$ touch each other at $\left(1,0,0,-\frac{1}{2}\right)$. Consider the point $I(x)$ on the edge $E_{0} E_{3}$ where the horoballs

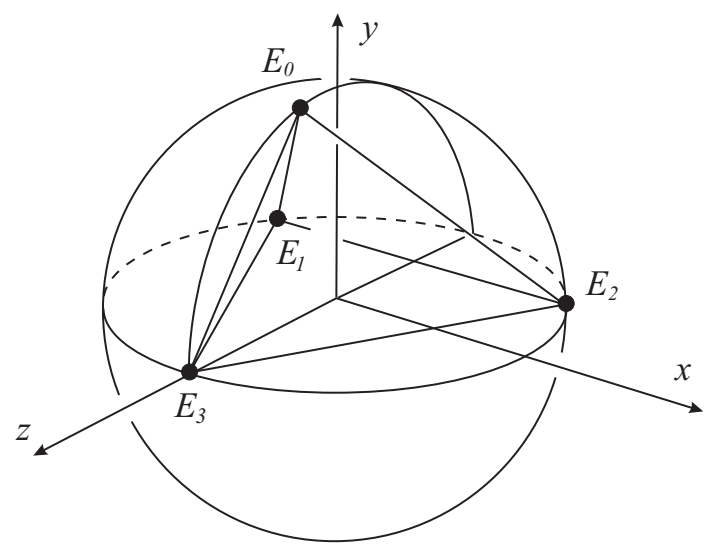

Figure 3:

$B_{i},(i=0,3)$ are tangent. Here $x$ denotes the hyperbolic distance between $I(0)$ and $I(x)$. Let $I\left(x_{1}\right) \in E_{0} E_{3}$ be such a point and parameter $x_{1}$ where 
horoball $B_{3}\left(x_{1}\right)$ touches $B_{2}(0)$ and $B_{1}(0)$. This horoball arrangement is denoted by $\mathcal{B}_{1}\left(x_{1}(z)\right)$. Function $V_{1}(x)$ to $\mathcal{B}_{1}$ is defined by:

$$
V_{1}(x):=\frac{1}{2} V(0)+\frac{1}{4} V(0) e^{2 x}+\frac{1}{4} V(0) e^{-2 x}, \quad x \in\left(0, x_{1}(z)\right] .
$$

By Lemma 3.1 it follows that function $V_{1}(x)$ strictly increases as $I(x)$ ( $x \in$ $\left.\left(0, x_{1}(z)\right]\right)$ moves away from $I(0)$ along $E_{0} E_{3}$. That means that the density function for any $z \in\left(0, \frac{-2}{13}+\frac{6 \sqrt{3}}{13}\right],\left(\frac{-2}{13}+\frac{6 \sqrt{3}}{13} \approx 0.64556191\right)$

$$
\delta\left(\mathcal{B}_{1}(x(z))\right)=\frac{V_{1}(x)}{\operatorname{vol}(T(\alpha))}
$$

attains its maximum at the endpoint $x_{1}$. Such a configuration does not occur if $z \in\left(\frac{-2}{13}+\frac{6 \sqrt{3}}{13}, 1\right]$.

To study the former density function $\delta\left(\mathcal{B}_{1}\left(x_{1}(z)\right)\right)$ we have to compute the volume sum of the horoball sectors $V_{1}\left(x_{1}\right)=\Sigma_{1}^{4}\left(B_{i} \cap T(\alpha)\right)$ belonging to the parameter $x_{1}$. Of course the volume $V_{1}\left(x_{1}\right)$ and the volume of the tetrahedron $T(\alpha)$ depend on parameter $z$. First we calculate the six intersection points of the edges of $T(\alpha)$ and horoballs $B_{i}(i=0,1,2,3)$, using the above projective coordinate system and the equations of the horospheres derived from (2.7). These intersection points on the edge $E_{i} E_{j}$ is denoted by $M_{i j}^{1}$ $(i<j), i, j \in\{0,1,2,3\}$.

$$
\begin{gathered}
M_{03}^{1}=\left(1,0,-3 \frac{\sqrt{1-z^{2}}}{2 z-5},-\frac{z+2}{2 z-5}\right), M_{13}^{1}=\left(1,-\frac{\sqrt{3}}{4}, 0, \frac{1}{4}\right), \\
M_{23}^{1}=\left(1, \frac{\sqrt{3}}{4}, 0, \frac{1}{4}\right), M_{12}^{1}=\left(1,0,0,-\frac{1}{2}\right) \\
M_{02}^{1}=\left(1, \frac{\sqrt{3}(z+2)}{2(z+5)}, 3 \frac{\sqrt{1-z^{2}}}{z+5}, \frac{5 z-2}{2(z+5)}\right), \\
M_{01}^{1}=\left(1,-\frac{\sqrt{3}(z+2)}{2(z+5)}, 3 \frac{\sqrt{1-z^{2}}}{z+5}, \frac{5 z-2}{2(z+5)}\right) .
\end{gathered}
$$

The volumes of horoball sectors (depending on parameter $z$ ) can be computed by Bolyai's formulas (2.8-9) and the volume of the tetrahedron $T(\alpha)$ is determined by formula (3.1). Finally, we obtain the density function $\delta\left(\mathcal{B}_{1}\left(x_{1}(z)\right)\right)=\frac{V_{1}\left(x_{1}\right)}{\operatorname{vol}(T(\alpha))}$ which depends only on parameter $z \in\left(0, \frac{-2}{13}+\right.$ $\left.\frac{6 \sqrt{3}}{13}\right]$. By careful computer analysis of the above density function we get 
that the function is convex, it attains its maximum at the upper endpoint of the interval $\left(0, \frac{-2}{13}+\frac{6 \sqrt{3}}{13}\right]$. We have studied the above function with Maple using that the conditions of the Lobachevsky function are well known [22]. The graph of $\delta\left(\mathcal{B}_{1}\left(x_{1}(z)\right)\right)$ can be seen in Fig. 4. Note, that the case

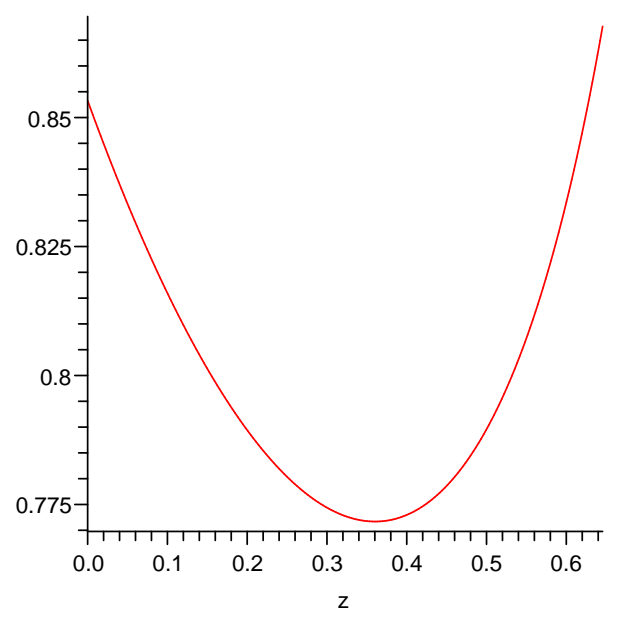

Figure 4:

$z=0$ belongs to the optimal arrangement of Böröczky-Florian, its density is $\approx 0.85327609$. The density at the upper endpoint of the interval $\left(0, \frac{-2}{13}+\frac{6 \sqrt{3}}{13}\right]$ is larger than that, namely:

$$
\delta\left(\mathcal{B}_{1}\left(x_{1}\left(\frac{-2}{13}+\frac{6 \sqrt{3}}{13}\right)\right)\right) \approx 0.86767481, \quad(\alpha \approx 1.30899694) .
$$

b.) We consider the former horoball arrangement $\mathcal{B}_{1}\left(x_{1}(z)\right)$ for any $z \in\left(0, \frac{-2}{13}+\right.$ $\left.\frac{6 \sqrt{3}}{13}\right]$. We expand the above "larger horoball" $B_{3}\left(x_{1}\right)$ until it touches the opposite face $E_{0} E_{1} E_{2}$ of the tetrahedron while keeping the other horoballs tangent to it. The arising horoball arrangement are realizable for any $z \in$ $\left(0, \frac{-2}{13}+\frac{6 \sqrt{3}}{13}\right]$.

Similarly to the case a.) we consider a point $I(x)\left(x \geq x_{1}\right)$ (on the edge $E_{0} E_{3}$ where the horoballs $B_{i},(i=0,3)$ are tangent at point $I(x) \in E_{0} E_{3}$. Let $x-x_{1}$ denote the hyperbolic distance between $I\left(x_{1}\right)$ and $I(x)$. Furthermore, let $I\left(x_{2}\right) \in E_{0} E_{3}$ be such a point where horoball $B_{3}\left(x_{2}\right)$ of parameter 
$x_{2}$ touches the face $E_{0} E_{1} E_{2}$. The "largest horoball" determines the configuration of all other horoballs and this horoball arrangement is denoted by $\mathcal{B}_{2}\left(x_{2}(z)\right)$. Function $V_{2}(x)$ is defined as follows:

$$
V_{2}(x):=\frac{1}{2} V(0) e^{-2\left(x-x_{1}\right)}+\frac{1}{4} V(0) e^{2 x}+\frac{1}{4} V(0) e^{-2 x}, x \in\left[x_{1}, x_{2}\right] .
$$

The following Lemma is obtained by examining the above function:

Lemma 3.2 The maxima of function $V_{2}(x)$ are realized to parameters $x_{1}$ or $x_{2}$.

By the above Lemma 3.2 it follows that it is sufficient to consider the volume function $V_{2}(x)$ and the density function $\delta\left(\mathcal{B}_{2}(x(z))\right)$ at the parameters $x_{1}$ and $x_{2}$. The case $x_{1}$ was taken in a.) thus we have to consider only the case $x=x_{2}$.

The horoball $B_{3}\left(x_{2}\right)$ has to touch the side face $E_{0} E_{1} E_{2}$ of fully asymptotic tetrahedron $T(\alpha)$. Thus, it is passing trough the foot point $E_{3}^{\prime}$ perpendicularly dropped from the point $E_{3}$ on the plane $E_{0} E_{1} E_{2}$,

$$
E_{3}^{\prime}=\left(1,0, \frac{3 \sqrt{\left(1-z^{2}\right)}(1+2 z)}{(z+5)^{2}},-\frac{7 z^{2}+4 z-2}{2 z^{2}-4 z-7}\right) .
$$

To examine the density function $\delta\left(\mathcal{B}_{2}\left(x_{2}(z)\right)\right)$ we have to compute the sum of the volumes of the horoball sectors $V_{2}\left(x_{2}\right)=\Sigma_{1}^{4}\left(B_{i} \cap T(\alpha)\right)$ to the parameter $x_{2}$. The volume $V_{2}\left(x_{2}\right)$ and the volume of the tetrahedron $T(\alpha)$ depend on parameter $z$. Similarly to the case a.) we have to determine the seven intersection points of the edges of $T(\alpha)$ and horoballs $B_{i}(i=$ $0,1,2,3)$. The intersection points on the edges $E_{0} E_{1}, E_{0} E_{2}, E_{0} E_{3}, E_{3} E_{2}$ and $E_{3} E_{1}$ are denoted by $M_{i j}^{2}(i<j), i, j \in\{0,1,2,3\}$. On the edge $E_{1} E_{2}$ there are no touching point but there are two point of intersections $M_{12,2}^{2}$.

$$
\begin{gathered}
M_{03}^{2}=\left(1,0,18 \frac{\sqrt{1-z^{2}}(1+z)}{4 z^{2}+22 z+22}, \frac{19 z^{2}+22 z+4}{4 z^{2}+22 z+22}\right), \\
M_{13}^{2}=\left(1,-\frac{6 \sqrt{3}\left(z^{2}-1\right)}{11 z^{2}-4 z-16}, 0,-\frac{7 z^{2}+4 z-2}{11 z^{2}-4 z-16}\right), \\
M_{23}^{2}=\left(1, \frac{6 \sqrt{3}\left(z^{2}-1\right)}{11 z^{2}-4 z-16}, 0,-\frac{7 z^{2}+4 z-2}{11 z^{2}-4 z-16}\right), \\
M_{12_{1,2}}^{2}=\left(1, \pm \frac{\sqrt{3}\left(13 z^{2}+4 z-8\right)}{2\left(11 z^{2}-4 z-16\right)}, 0,-\frac{1}{2}\right),
\end{gathered}
$$




$$
\begin{gathered}
M_{02}^{2}=\left(1, \frac{2 \sqrt{3}\left(z^{2}-1\right)}{4 z^{2}-z-6},-\frac{\sqrt{1-z^{2}}(z+2)}{4 z^{2}-z-6},-\frac{3 z^{2}+2 z-2}{4 z^{2}-z-6}\right), \\
M_{01}^{2}=\left(1,-\frac{2 \sqrt{3}\left(z^{2}-1\right)}{4 z^{2}-z-6},-\frac{\sqrt{1-z^{2}}(z+2)}{4 z^{2}-z-6},-\frac{3 z^{2}+2 z-2}{4 z^{2}-z-6}\right) .
\end{gathered}
$$

The volumes of horoball sectors (depending on parameter $z$ ) can be computed by Bolyai's formulas (2.8-9) and the volume of the tetrahedron $T(\alpha)$ is determined by formula (3.1). We get the density function $\delta\left(\mathcal{B}_{2}\left(x_{2}(z)\right)\right)=$ $\frac{V_{2}\left(x_{2}\right)}{\operatorname{vol}(T(\alpha))}$ which depend only on parameter $z \in\left(0, \frac{-2}{13}+\frac{6 \sqrt{3}}{13}\right]$. By careful investigation (see [22]) of the above density function we get that the function attains its maximum at the point $z=\frac{-2}{13}+\frac{6 \sqrt{3}}{13}$. The graph of $\delta\left(\mathcal{B}_{2}\left(x_{2}(z)\right)\right)$ is shown in Fig. 5. The density at the point $\frac{-2}{13}+\frac{6 \sqrt{3}}{13}$ is larger than the

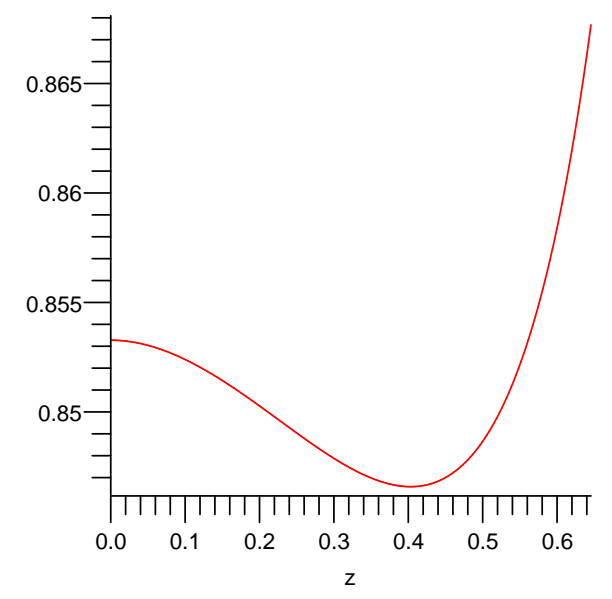

Figure 5:

Böröczky-Florian upper density, namely

$$
\delta\left(\mathcal{B}_{2}\left(x_{2}\left(\frac{-2}{13}+\frac{6 \sqrt{3}}{13}\right)\right)\right) \approx 0.86767481, \quad(\alpha \approx 1.30899694) .
$$

The horoball density of of Böröczky-Florian belongs to the parameter $z=$ 0 . To the parameter $z=\frac{-2}{13}+\frac{6 \sqrt{3}}{13}$ we get the same horoball density as in case a.).

i/2: $\left(z \in\left(\frac{-2}{13}+\frac{6 \sqrt{3}}{13}, 1\right]\right)$ 


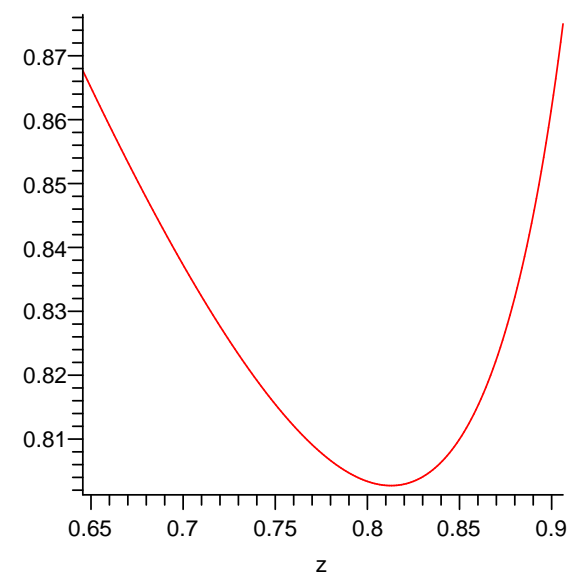

Figure 6:

a.) $z \in\left(\frac{-2}{13}+\frac{6 \sqrt{3}}{13}, z_{3}\right]$, where $z_{3}:=\frac{4}{73} \sqrt{3} \sqrt{178} \cos \left(\frac{1}{3} \arctan \left(\frac{73}{8001} \sqrt{3} \sqrt{229}\right)\right)-$ $\frac{26}{73} \approx 0.9061774494$.

In the former case $\mathbf{i} / \mathbf{1} / \mathbf{b}$ we have already considered the ball arrangement $\mathcal{B}_{1}\left(x_{1}(z)\right)$ for any $z \in\left(0, \frac{-2}{13}+\frac{6 \sqrt{3}}{13}\right]$. There we have expanded the "larger horoball" $B_{3}\left(x_{1}\right)$ until it touches the opposite face $E_{0} E_{1} E_{2}$ of the tetrahedron while keeping other horoballs tangent to it. But if $z \in\left(\frac{-2}{13}+\right.$ $\left.\frac{6 \sqrt{3}}{13}, z_{3}\right]$ then we cannot apply this procedure. Namely, if the horoball $B_{3}\left(x_{2}\right)$ touches the opposite side face, then $B_{3}\left(x_{2}\right)$ does not touch $B_{1}(0)$ and $B_{2}(0)$. Then we expand the horoball $B_{2}$ until it touches $B_{3}\left(x_{2}\right)$ or the opposite side face. This means that the "larger horoball" $B_{3}\left(x_{2}\right)$ touches the opposite face $E_{0} E_{1} E_{2}$ at point $E_{3}^{\prime}$ and the horoballs $B_{2}, B_{0}$ touch $B_{3}$. Then $B_{1}$ touches only the horoball $B_{2}$. This configuration occurs until the horoball $B_{2}$ is not tangent to its opposite face $E_{3} E_{1} E_{0}$.

This arrangement exactly exists if $z \in\left(\frac{-2}{13}+\frac{6 \sqrt{3}}{13}, z_{3}\right]$. The horoball $B_{2}$ touches the opposite side face $E_{0} E_{1} E_{3}$ of $T(\alpha)$ at the point $E_{2}^{\prime}$ :

$$
E_{2}^{\prime}=\left(\frac{5 z+7}{2(z+2)},-\frac{\sqrt{3}(2 z+1)}{2(z+2)}, \frac{3 \sqrt{1-z^{2}}}{2(z+2)}, \frac{2 z+1}{2(z+2)}\right) .
$$

Similarly to the case a.) the "largest horoball" determines the configuration of all other horoballs and this horoball arrangement is denoted by $\mathcal{B}_{3}\left(x_{3}(z)\right)$. 
The function $V_{3}\left(x_{3}(z)\right)$ and related density function

$$
\delta\left(\mathcal{B}_{3}\left(x_{3}(z)\right)\right)=\frac{V_{3}\left(x_{3}(z)\right)}{\operatorname{vol}(T(\alpha))}
$$

can be analysed in the same way as in $\mathbf{i} / \mathbf{1} / \mathbf{a}$ and $\mathbf{i} / \mathbf{1} / \mathbf{b}$ and we obtain that the convex density function attains its maximum at the above $z_{3}$.

The graph of $\left.\delta\left(\mathcal{B}_{3}\left(x_{3}, z\right)\right), z \in\left[\frac{-2}{13}+\frac{6 \sqrt{3}}{13}\right), z_{3}\right]$ is shown in the Fig. 6. While Fig. 7 shows the larger horoballs $B_{3}, B_{2} \in \mathcal{B}_{3}\left(x_{3}\left(z_{3}\right)\right)$ with tetrahedron $T(\alpha)$ in the Beltrami-Cayley-Klein model. The density at the point $z=z_{3}$

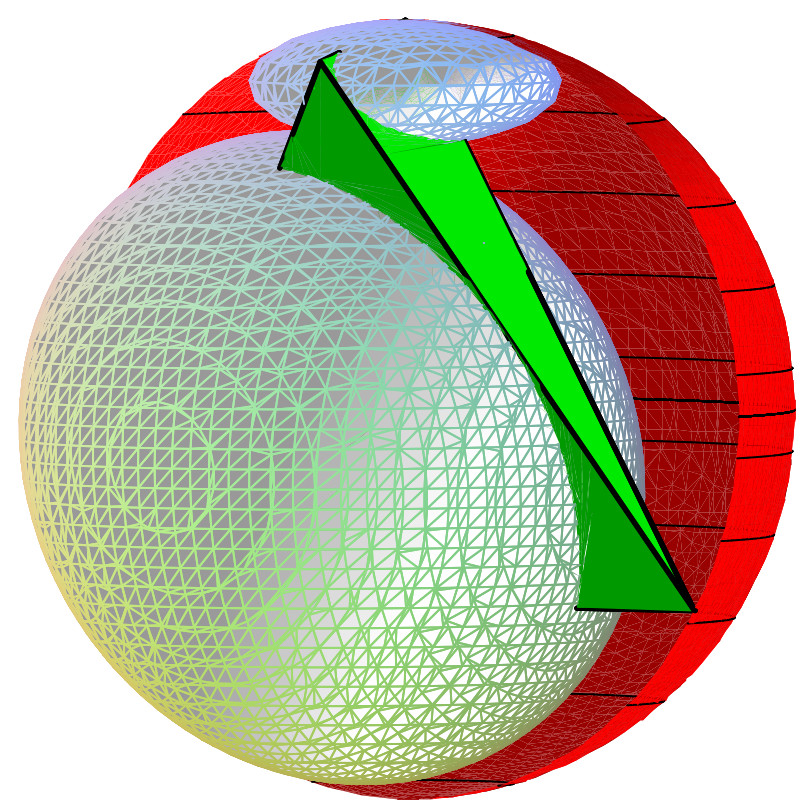

Figure 7: The optimal horoball packing arrangement related to a fully asymptotic tetrahedron

is larger than the above maxima

$$
\delta\left(\mathcal{B}_{3}\left(x_{3}\left(z_{3}\right)\right)\right) \approx 0.87499429, \quad(\alpha \approx 1.44340117) .
$$

b.) $z \in\left(z_{3}, 1\right)$

We consider such ball arrangements $\mathcal{B}_{4}\left(x_{4}(z)\right)$ where the "larger horoballs" $B_{3}$ and $B_{2}$ touch their opposite faces $E_{0} E_{1} E_{2}$ and $E_{0} E_{1} E_{3}$, respectively. The horoballs $B_{0}, B_{3}$ and $B_{1}, B_{2}$ pairwise touch each other. This horoball 
packing can be studied similarly to the above cases and we can analyse the density function $\delta\left(\mathcal{B}_{4}\left(x_{4}(z)\right)\right.$ ) (see Fig. 8), not detailed further. We obtain, that the density function is stricly decreasing in the interval $\left(z_{3}, 1\right)$. Thus, there is no horoball arrangement with larger density than $\mathcal{B}_{3}\left(x_{3}\left(z_{3}\right)\right)$ with density $\approx 0.87499429$ (see (3.7)).

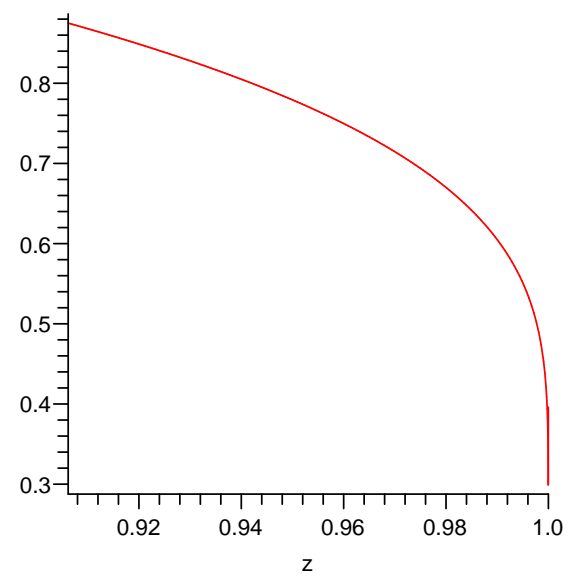

Figure 8:

Analogously to (i) we have investigated the cases (ii) $(-1<z<0)$, not detailed more, and we have obtained the following

Theorem 3.3 The densest horoball arrangement, related to the generalized simplicial density function (Definition 1.3) belongs to the horoball arrangement $\mathcal{B}_{3}\left(x_{3}\left(z_{3}\right)\right.$ ) (see (3.7) and Fig. 7) with density

$$
\delta\left(\mathcal{B}_{3}\left(x_{3}\left(z_{3}\right)\right)\right) \approx 0.87499429 .
$$

Remark 3.4 This packing seems not to have global extension to the entire hyperbolic space $\overline{\mathbf{H}}^{3}$, so that the same density occurs in each asymptotic tetrahedron. We plan to investigate this problem with E. Molnár and I. Prok by [12].

Optimal sphere packings in other homogeneous Thurston geometries represent a class of open mathematical problems. For these non-Euclidean geometries only very few results are known [20], [21]. Detailed studies are the objective of ongoing research. 


\section{References}

[1] Bezdek, K. Sphere Packings Revisited, European Journal of Combinatorics (2006) 27/6 , 864-883.

[2] Bowen, L. - Radin, C. Optimally Dense Packings of Hyperbolic Space, Geometriae Dedicata, (2004) 104, 37-59.

[3] Böhm, J. - Hertel, E. Polyedergeometrie in n-dimensionalen Räumen konstanter Krümmung, Birkhäuser, Basel (1981).

[4] Böröczky, K. Packing of spheres in spaces of constant curvature, Acta Math. Acad. Sci. Hungar., (1978) 32 , 243-261.

[5] Böröczky, K. - Florian, A. Über die dichteste Kugelpackung im hyperbolischen Raum, Acta Math. Acad. Sci. Hungar., (1964) 15 , 237-245.

[6] Coxeter, H. S. M. Regular honeycombs in hyperbolic space, Proceedings of the international Congress of Mathematicians, Amsterdam, (1954) III , 155-169.

[7] Fejes Tóth, G. - Kuperberg, G. - Kuperberg, W. Highly Saturated Packings and Reduced Coverings, Monatshefte für Mathematik, (1998) 125/2, 127-145.

[8] Kellerhals, R. Ball packings in spaces of constant curvature and the simplicial density function, Journal für reine und angewandte Mathematik, (1998) 494, 189-203.

[9] Kozma, T. R. - Szirmai, J. Optimally dense packings for fully asymptotic Coxeter tilings by horoballs of different types, Submitted to Monatshefte für Mathematik, 2011.

[10] Molnár, E. Klassifikation der hyperbolischen Dodekaederpflasterungen von flächentransitiven Bewegungsgruppen, Mathematica Pannonica , (1993) 4/1, 113-136.

[11] Molnár, E. The projective interpretation of the eight 3-dimensional homogeneous geometries, Beiträge Alg. Geom., (Contr. Alg. Geom.), (1997) 38/2, 261-288. 
[12] Molnár, E. - Prok, I. - Szirmai, J. Classification of tile-transitive 3-simplex tilings and their realizations in homogeneous spaces. A. Prékopa and E. Molnár, (eds.). Non-Euclidean Geometries, János Bolyai Memorial Volume, Mathematics and Its Applications, Springer (2006) Vol. 581, 321-363.

[13] Marshall, T. H. Asymptotic Volume Formulae and Hyperbolic Ball Packing, Annales Academia Scientiarum Fennica: Mathematica, (1999) 24, 31-43.

[14] Milnor, J. Geometry, Collected papers, Publish or Perish, (1994) Vol 1.

[15] Radin, C. The symmetry of optimally dense packings, NonEucledian Geometries, eds. A. Prekopa, E. Molnar, pp. 197-207, Springer Verlag, (2006).

[16] Rogers, C. A. Packing and covering, Cambridge University Press, (1964).

Szirmai, J. Horoball packings for the Lambert-cube tilings in the hyperbolic 3-space, Beiträge Alg. Geom., (Contr. Alg. Geom.), (2005) 46(1), 43-60.

Szirmai, J. The optimal ball and horoball packings of the Coxeter tilings in the hyperbolic 3-space Beiträge Alg. Geom., (Contr. Alg. Geom.), (2005) 46/2, 545-558.

Szirmai, J. The optimal ball and horoball packings to the Coxeter honeycombs in the hyperbolic d-space Beiträge Alg. Geom., (Contr. Alg. Geom.), (2007) 48/1, 35-47.

[20] Szirmai, J. The densest geodesic ball packing by a type of Nil lattices Beiträge Alg. Geom., (Contr. Alg. Geom.), (2007) 48/2, 383397.

[21] Szirmai, J. The densest translation ball packing by fundamental lattices in Sol space Beiträge Alg. Geom., (Contr. Alg. Geom.), (2010) $\mathbf{5 1 / 2}, 353-373$. 
Horoball packings and their densities...

[22] Zagier, D. The Dilogarithm Function Frontiers in Number Theory, Physics, and Geometry II : On Conformal Field Theories, Discrete Groups and Renormalization, Springer, (2007) 3-65. 